gr-qc/9309026

\title{
Black Hole Entropy and the
}

\section{Dimensional Continuation of the Gauss-Bonnet Theorem}

\author{
Máximo Bañados ${ }^{1}$, Claudio Teitelboim ${ }^{1,2}$ and Jorge Zanelli ${ }^{1,3}$ \\ ${ }^{1}$ Centro de Estudios Científicos de Santiago, Casilla 16443, Santiago 9, Chile. \\ ${ }^{2}$ Institute for Advanced Study, Olden Lane, Princeton, New Jersey 08540. \\ ${ }^{3}$ Departamento de Física, Facultad de Ciencias, Universidad de Chile, \\ Casilla 653, Santiago, Chile.
}

\begin{abstract}
04.50.+h, 05.70.Ce, 97.60.Lf
\end{abstract}
Abstract

Typeset using REVTEX 
The purpose of this note is to point out that the black-hole entropy may be derived in a simple manner if one regards the Euclidean Hilbert action for the gravitational field as the dimensional continuation of a topological invariant, the Euler class. Then, the dimensional continuation of the Gauss-Bonnet theorem shows that the entropy itself is the continuation of the Euler class of a small disk centered at the horizon.

The Euclidean spacetimes admitted in the action principle will have the topology $\Re^{2} \times$ $\mathcal{S}^{d-2}$. We allow for cusps at any point in $\Re^{2}$. The deficit angle of a cusp at a given point turns out to be canonically conjugate to the area of the $\mathcal{S}^{d-2}$ at that point. In particular, the entropy is canonically conjugate to the deficit angle at the horizon. The condition for zero deficit angle emerges from extremizing the action with respect to the area of the $\mathcal{S}^{d-2}$. Thus, the black hole temperature is determined, in the semiclassical approximation, by extremizing with respect to the area of the $\mathcal{S}^{d-2}$ at the horizon (summing over all horizons in the path integral).

This result applies also to the natural generalization of the Hilbert action to higher spacetime dimensions, the Lovelock action [1]. This action, which keeps the field equations for the metric of second order and hence does not change the degrees of freedom, can also be understood in terms of dimensional continuation [2, 3]. For a spacetime of dimension $d$, the generalized action contains the dimensionally continued Euler classes of all even dimensions $2 p<d$. Each such term gives rise to an entropy [四 that is proportional to a dimensional continuation of the Euler class of dimension $2 p-2$. Thus, the Hilbert action with a cosmological constant may be thought of as coming from dimensions $2 p=2$ and $2 p=0$, respectively. The entropy comes then from $2 p=0$. (We define the Euler class for a space of dimension zero as unity and that for a space of negative dimension as zero.)

We will first recall the Gauss-Bonnet theorem and bring out its relationship with the Hilbert action for the gravitational field. The extension to the Lovelock theory will be indicated at the end.

If one considers a two dimensional manifold $M$ with boundary $\partial M$, the Gauss-Bonnet theorem reads 


$$
\frac{1}{2} \int_{M} \sqrt{g} g^{\mu \nu} R_{\mu \alpha \nu}^{\alpha} d^{2} x-\int_{\partial M} \sqrt{g} K d^{1} x=2 \pi \chi(M)
$$

The integer $\chi(M)$ on the right hand side of (1) is the Euler number of $M$ and depends solely on its topology. One has $\chi=1$ for a disk and $\chi=0$ for an annulus. We will refer to the sum of integrals appearing on the left side of (11) as the Euler class of $M$. The Gauss-Bonnet theorem then says that the Euler class of $M$ is equal to $2 \pi$ times its Euler number.

If one varies the integral over $M$ in (1) one finds, by virtue of the Bianchi identity, that the piece coming from the variation of the Riemann tensor yields a surface term. This surface term exactly cancels the variation of the surface integral appearing in the Euler class. On the other hand, because of the special algebraic properties of the Riemann tensor in two spacetime dimensions, the contribution of the variation of $\sqrt{g} g^{\mu \nu}$ is identically zero. This is a poor man's way to put into evidence that the Euler class is "a topological invariant", the real work is to show that the actual value of the sum of integrals is $2 \pi \chi$.

Now, the Hilbert action for the gravitational field in $d$ Euclidean spacetime dimensions may be written as

$$
I_{H}=\frac{1}{2} \int_{M} \sqrt{g} g^{\mu \nu} R_{\mu \alpha \nu}^{\alpha} d^{d} x-\int_{\partial M} \sqrt{g} K d^{d-1} x
$$

[One integrates $\exp (+I)$ in the Euclidean path integral.]

This action has the same form as the Euler class of two dimensions, with the change that now the integrals, and the geometric expressions appearing in them, refer to a spacetime of dimension $d>2$. For this reason, one says that the Hilbert action is the dimensional continuation of the Euler class of two dimensions. After dimensional continuation, the Euler class ceases to be a topological invariant. While it is still true that the variation of the Riemann tensor in (2) yields a surface term, this surface term no longer cancels the variation of the integral of the extrinsic curvature. Rather, the sum of the two variations vanishes only when the intrinsic geometry of the boundary is held fixed. Moreover, the contribution to the variation coming from $\sqrt{g} g^{\mu \nu}$ gives the Einstein tensor, which is no longer identically zero, and hence the demand that it vanishes is not empty but gives the Einstein equations. 
There is another action, which differs from the $I_{H}$ by a boundary term. It is the canonical action

$$
I_{C}=\int\left(\pi^{i j} \dot{g}_{i j}-N \mathcal{H}-N^{i} \mathcal{H}_{i}\right)
$$

When one studies black holes $I_{C}$ has a significant advantage over the Hilbert action. It vanishes on the black hole due to the constraint equations $\mathcal{H}=0=\mathcal{H}_{i}$ and the time independence of the spatial metric. The black hole entropy and its relation with the GaussBonnet theorem will arise through the difference between the Hilbert and the canonical actions.

In the Euclidean formalism for black holes, it is useful to introduce in the $\Re^{2}$ factor of $\Re^{2} \times \mathcal{S}^{d-2}$, a polar system of coordinates. The reason is that the black hole will have a Killing vector field -the Killing time- whose orbits are circles centered at the horizon. But, it should be stressed that the discussion that follows is valid for a system of polar coordinates centered anywhere in $\Re^{2}$. Indeed the Killing vector exists only on the extremum and not for a generic spacetime admited in the action principle.

Take now a polar angle in $\Re^{2}$ as the time variable in a Hamiltonian analysis. An initial surface of time $t_{1}$ and a final surface of time $t_{2}$ will meet at the origin, which is a fixed point of the time vector field. There is nothing wrong with the two surfaces intersecting. The hamiltonian formalism can handle that.

Consider now in $\Re^{2}$ a small disk $D_{\epsilon}$ of radius $\epsilon$ around the origin. The portion of spacetime between $t_{1}$ and $t_{2}$ that remains inside the small disk is a triangle $\triangle_{\epsilon}$, of angular opening $t_{2}-t_{1}$, to each point of which an $\mathcal{S}^{d-2}$ sphere is attached. The action (3) for $\triangle_{\epsilon} \times \mathcal{S}^{d-2}$ tends to zero if one lets $\epsilon \rightarrow 0$ because the integrand is smooth. On the other hand, the covariant action (2) tends to a non-zero limit - which will be exhibited in Eq. (7) below. Thus we have

$$
\begin{aligned}
& \lim _{\epsilon \rightarrow 0} I_{C}\left[\triangle_{\epsilon} \times \mathcal{S}^{d-2}\right]=0, \\
& \lim _{\epsilon \rightarrow 0} I_{H}\left[\triangle_{\epsilon} \times \mathcal{S}^{d-2}\right] \neq 0 .
\end{aligned}
$$


Next, recall that the very derivation of the canonical action (see for example [3]), shows that for surfaces of constant $t$, the inclusion of the extrinsic curvature term precisely turns (2) into (3). Combining this fact with the preceding remarks we see that for the whole region between $t_{1}$ and $t_{2}$ we have

$$
I_{H}=\lim _{\epsilon \rightarrow 0} I_{H}\left[\triangle_{\epsilon} \times \mathcal{S}^{d-2}\right]+I_{C}+B_{\infty}
$$

Here $B_{\infty}$, which needs not be explicitly written, stands for a surface term over a large circle in $\Re^{2} \times \mathcal{S}^{d-2}$. Indeed, as stated above, the Hilbert action (2) needs the intrinsic geometry of the entire boundary to be fixed. On the other hand for the Hamiltonian action (3) one must fix at $t_{1}$ and $t_{2}$ the intrinsic geometries of those boundaries -including their intersection at the origin- and, at infinity, the mass $M$ and angular momentum $J$-with a precise rate of fall off for the fields [see, for example [5]]. If instead of $M$, one fixes its conjugate, the asymptotic Killing time difference $t_{2}-t_{1}=\beta$, while still keeping $J$ fixed, one must substract $\beta M$ from (3). Thus, if we drop $B_{\infty}$, we obtain the improved covariant action,

$$
I=\lim _{\epsilon \rightarrow 0} I_{H}\left[\triangle_{\epsilon} \times \mathcal{S}^{d-2}\right]+I_{C},
$$

which is suited for fixing the intrinsic geometries of the surfaces at $t_{1}$ and $t_{2}$, and $M$ and $J$ at infinity. The action (6) differs from expression (5) only by a local surface term at infinity due to the different boundary condition there, and it is therefore as covariant as (2). Furthermore, (6) is finite on the black hole and thus it is "already regularized". [The Hilbert action (2) is infinite on the black hole because $B_{\infty}$ diverges.]

A short analysis reveals that the first term in (6) factorizes into the product of the Euler class (四) for $\triangle_{\epsilon}$ and the area of the $\mathcal{S}^{d-2}$ at the origin. But, the triangle $\triangle_{\epsilon}$ is topologically a disk and hence its Euler class is equal to $2 \pi$. Thus one finds

$$
\lim _{\epsilon \rightarrow 0} I_{H}\left[\triangle_{\epsilon} \times \mathcal{S}^{d-2}\right]=2 \pi \times\left(\text { area of } \mathcal{S}^{d-2}\right)_{\text {origin }}
$$

It is of interest to allow in (6) for a "cusp of deficit angle $\alpha$ " at the origin of $\Re^{2}$. This means that the value of the two-dimensional integral in the Euler class (1) is equal to $\alpha$, 
whereas the line integral over the boundary has the value $2 \pi-\alpha$. The full action (6) depends on $\alpha$. This is most directly seen by recalling that -as stated in (5)- the action (6) differs from the Hilbert action (2) by a local boundary term at infinity. As a consequence, if the geometry of the $\mathcal{S}^{d-2}$ at the cusp is varied, while keeping the rest of the configuration unaltered, one finds that the action changes by

$$
\delta I=\alpha \delta\left(\text { area of } \mathcal{S}^{d-2} \text { at cusp }\right)
$$

Equation (8) shows that the deficit angle -which is a property of the intrinsic Riemannian geometry of $\Re^{2}-$, is canonically conjugate to the area of the $\mathcal{S}^{d-2}$ attached to that point -an extrinsic property.

Observe that one could incorrectly believe, due to (7), that the action (6) (and hence its variation) is independent of the deficit angle $\alpha$. What happens is that there is a boundary term in the variation of the canonical action, coming from space derivatives in $\mathcal{H}$, which cancels the variation of the surface term in the Euler class [6] leaving (8) as the net change.

As shown by (7), the actions (3) and (6) differ by a contact transformation which depends only on (part of) the common boundary data for each action. Thus, both actions correctly yield Einstein's equations and on this basis they are equally good. However, one wants to do more, one wants an action that can also be used to evaluate the partition function.

In the semiclassical approximation, the partition function is equal to the exponential of the classical action for a "closed Euclidean history". In the present case the closed Euclidean history is obtained by making the surface $t_{2}$ come around a full turn and coincide with the surface $t_{1}$. The triangle $\triangle_{\epsilon}$ becomes the whole disk $D_{\epsilon}$. One then extremizes with respect to the geometry of spacetime keeping the asymptotic data $M$ and $J$ fixed. For this problem, the improved action(6) and the canonical action (5) are not equivalent.

The black hole will be an extremum for the covariant action (6), because the demand that the variation (8) vanishes yields $\alpha=0$ at all points, which is the condition for the manifold to be metrically smooth. This is a property that the Euclidean black hole indeed posseses, since the empty space Einstein equations are obeyed everywhere. On the other hand, the 
demand that the canonical action should have an extremum with respect to variations of the area of the $\mathcal{S}^{d-2}$, would yield $\alpha=2 \pi$ at the origin, which would introduce a sort of source there.

Thus, adding the Hilbert action for a small disk around the origin to the canonical action restores covariance without introducing sources. This addition ensures that the fixed point can be located anywhere. This must be so since the manifold has only one boundary, that at infinity.

Note that the need for the contribution of $D_{\epsilon} \times \mathcal{S}^{d-2}$ in the action for the partition function, implies that the contribution (7) of $\triangle_{\epsilon} \times \mathcal{S}^{d-2}$ must already be included in the action for the transition amplitude from $t_{1}$ to $t_{2}$. This is because the partition function is the trace of the transition amplitude [7].

Consider now the value of the action on the extremum. Then it is convenient to take the polar angle to be the Killing time, for -in that case- the spatial geometry $g_{i j}$ is time independent. Furthermore, since the Hamiltonian contraints $\mathcal{H}=\mathcal{H}_{i}=0$ hold on the extremum, the value of the improved action (6) for the black hole is just the contribution of the disk at the horizon.

Since in (6) $M$ and $J$ are fixed, which corresponds to the microcanonical ensemble, we learn that the entropy is given by

$$
S=2 \pi \times\left(\text { area of } \mathcal{S}^{d-2}\right)_{\text {horizon }} .
$$

This is the standard expression for the black hole entropy in Einstein's theory. Note that the overall factor in front of the area, usually quoted as one fourth in units where Newton's constant is unity, is really the Euler class of the two-dimensional disk.

The preceding analysis goes through step by step for the Lovelock theory [1]. The analog of the Hilbert action given by (2) is

$$
I_{L}=\sum_{2 p<d} \frac{\alpha_{p}}{2^{2 p} p !}\left(I_{L}^{p}+B^{p}\right),
$$

with 


$$
I_{L}^{p}=\int_{M} \sqrt{g} \delta_{\left[\alpha_{1} \cdots \alpha_{2 p}\right]}^{\left[\beta_{1} \cdots \beta_{2 p}\right]} R_{\beta_{1} \beta_{2}}^{\alpha_{1} \alpha_{2}} \cdots R_{\beta_{2 p-1} \beta_{2 p}}^{\alpha_{2 p-1} \alpha_{2 p}} d^{d} x
$$

[Here the totally antisymmetrized Kronecker symbol is normalized so that it takes the values $0, \pm 1]$.

The boundary term $B^{p}$ is the generalization of the integrated trace of the extrinsic curvature in (2). It is given by

$$
B^{p}=\frac{-2}{d-2 p} \int_{\partial M} d^{d-1} x g_{i j} \pi_{(p)}^{i j}
$$

Here $\pi_{(p)}^{i j}$ is the contribution of (11) to the momentum canonically conjugate to the metric $g_{i j}$ of $\partial M$. It may be expressed as a function of the intrinsic and extrinsic curvatures of the boundary [3].

For Euclidean black holes in $d$-spacetime dimensions, again with topology $\Re^{2} \times \mathcal{S}^{d-2}$ [8], the action (6) now reads

$$
I=\lim _{\epsilon \rightarrow 0} I_{L}\left[D_{\epsilon} \times \mathcal{S}^{d-2}\right]+I_{C}
$$

and the entropy becomes

$$
S=\lim _{\epsilon \rightarrow 0} I_{L}\left[D_{\epsilon} \times \mathcal{S}^{d-2}\right]
$$

The limit (14) factorizes into the Euler class of the disk, equal to $2 \pi$, and a sum of dimensional continuations to $\mathcal{S}^{d-2}$ of the Euler classes of all even dimensions below $d-2$,

$$
S=2 \pi \times \sum_{2 p<d} \frac{\alpha_{p}}{2^{2(p-1)}[2(p-1)] !} S^{p-1}
$$

with

$$
S^{p}=\int \sqrt{g} \delta_{\left[\alpha_{1} \cdots \alpha_{2 p}\right]}^{\left[\beta_{1} \cdots \beta_{2 p}\right]} R_{\beta_{1} \beta_{2}}^{\alpha_{1} \alpha_{2}} \cdots R_{\beta_{2 p-1} \beta_{2 p}}^{\alpha_{2 p-1} \alpha_{2 p}} d^{d-2} x
$$

where the integral is taken over the $(d-2)$-sphere at the horizon.

The Hilbert action corresponds to $2 p=2$ and the corresponding entropy is $2 \pi$ times the area. The cosmological constant term corresponds to $2 p=0$ and gives no contribution to 
the entropy. Expression (15) was first given in [4].

The authors are grateful to Ted Jacobson and Robert Myers for urging them to prepare this analysis for publication. Appreciation is also extended to Steven Carlip and Marc Henneaux for many rewarding discussions. This work was partially supported by grants 0862/91, and 193.0910/93 from FONDECYT (Chile), by a European Communities research contract, by institutional support to the Centro de Estudios Científicos de Santiago provided by SAREC (Sweden) and a group of chilean private companies (COPEC, CMPC, ENERSIS). 


\section{REFERENCES}

[1] D. Lovelock, J. of Math. Phys. 12, 498 (1971).

[2] B. Zumino, Phys. Rep. 137, 108 (1986).

[3] C. Teitelboim and J. Zanelli, Class. and Quant.Grav. 4, L125 (1987); and in Constraint Theory and Relativistic Dynamics, G. Longhi and L. Lussana, eds. (World Scientific, Singapore, 1987).

[4] T. Jacobson and R. Myers, Phys. Rev. Lett. 70, 3684 (1993).

[5] T. Regge and C. Teitelboim, Ann.Phys. (N.Y.) 88, 286 (1974).

[6] See J.D. Brown and J.W. York, Phys. Rev. D. 47, 1420 (1993), for a lucid discussion of this point. These authors assume $\alpha=0$ from the outset and do not obtain it as an equation of motion. They do not discuss the role of the Gauss-Bonnet theorem.

[7] Note that the term which must be added to the canonical action does not refer to any history in time $t$. This is a deep property of the black-hole. Steven Carlip (private communication) describes it by saying that the action of the small triangle refers to "intrinsically time independent degrees of freedom".

[8] M. Bañados, C. Teitelboim and J.Zanelli, CECS/IAS-preprint (1992), gr-qc/9307033, to be published in Phys. Rev. D. 